\title{
Scars in Pediatric Patients
}

Anne Le Touze

\section{Contents}

\subsection{Introduction/Background - 398}

46.2 Healing Specificities in Children - 398

46.2.1 Fetal Healing - 398

46.2.2 Pediatric Peculiarities in Healing - 398

46.2.3 How to Manage Wound Healing in Children - 398

46.3 Pathological Scars - 399

46.3.1 Clinical and Histological Aspects - 399

46.3.2 Easing Factors - 400

46.3.3 Prevention and Treatment [13] - 401

46.4 Defective or Disgracious Scars - 403

46.5 Scars and Growth -403

46.6 Conclusion -403

References -403 


\subsection{Introduction/Background}

A child is not a miniature adult. Although physiological healing process is not different in children, their growth potential gives them some specificities in terms of healing and scaring. Disturbance of healing process or inappropriate wound treatment will lead to pathologic scar. Pathologic scars can disrupt growth in children.

\subsection{Healing Specificities in Children}

\subsubsection{Fetal Healing}

Experimental and clinical studies point that fetal healing is scarless [1]. Fetal healing mechanisms are still poorly known, although many endogenous and exogenous factors seem to differ from those of adult healing mechanisms. The main difference probably lies at the level of the immune system and the inflammatory response. All these studies concern different animal species and any extrapolation towards human species should be cautious.

Macrophages are known to be the major agents of the inflammatory response and tissue regeneration process in adults. In early gestation, healing process is accomplished with poor influx of macrophages, while the influx of macrophages in the wound is more important at the end of gestation [2]. The embryo seems to be able to heal without macrophages, and therefore without undergoing inflammatory response. Nevertheless, the embryo is able to respond to inflammatory stimuli [3]. Explanation for this paradox could be the immaturity of the inflammatory system, but we need further exploration to understand this ambiguity.

Collagen is scarce in the healing wound due to the lack of response of the fibroblasts to transforming growth factor beta 1 (TGF $\beta 1$ ). TGF $\beta 1$ induces glycosaminoglycan synthesis all along gestation: glycosaminoglycan is much more important than collagen in the scar matrix in fetus [4].

Amniotic fluid provides the fetus with an environment rich in growth factors necessary for its development. High concentration of hyaluronic acid in the amniotic fluid has been supposed to be the extrinsic and intrinsic factor of non-inflammatory fetal healing [5]. Further experimental studies need to prove that the quality of fetal wound healing is due to intrinsic factors [6]. Even if scarless fetal healing mechanisms are uncertain, absence of inflammatory response seems to be the key point.

Nevertheless, at the end of gestation, inflammatory response appears and scar becomes visible. Further exploration of fetal healing and better knowledge should allow for promising clinical applications.

\subsubsection{Pediatric Peculiarities in Healing}

Very few data on this subject is available in the literature. Nevertheless, "healing behavior" is peculiar and varies all along childhood [7].

- 0-6 months

Infants before the age of 6 months heal very fast and with very discreet scar. It happens as if the inflammatory response was immature, therefore attenuated. Perhaps some phenomena of prenatal period are perpetuated after birth.

- 2 years - teenage

On the contrary, the inflammatory expression of the scar dominates this period, and the phenomena of remodeling seem amplified, major, and disabling. The few comorbidities of healthy organisms and the physiological mechanisms linked to growth are an undeniable asset for the good evolution of the child's wounds. Scarring is often very fast but can be explosive. Hypertrophic scaring could be considered as physiological at this age, as it is constant.

- 6 months -2 years

The evolutive pattern of scar is unpredictable at this age, sometimes still discreet, sometimes already very inflammatory.

\subsubsection{How to Manage Wound Healing in Children}

As the inflammatory response is constant and "explosive," the healing process must be carried out over a short period of time to prevent excessive production of collagen.

Except for children under palliative care, most of the wounds can undergo surgery: mechanical cleansing, suturing, grafting, flaps. Before, instead of or after surgery, we use dressings or negative pressure wound therapy (NPWT).

Management of pain is essential and difficult: pain and fear are often entangled; level 2 painkillers are not recommended before 12 years old. Therefore, level 3 painkillers are often necessary but to be administrated in health institutions. Inhalation of $50-50 \% \mathrm{~N}_{2} \mathrm{O}-\mathrm{O}_{2}$ mixture and any adjuvant technique of analgesia may be useful (hypnoanalgesia, video distraction, virtual reality, etc.) $[8,9]$.

Surgical techniques have to be adapted according to age, psychomotor development, thickness of teguments, and potential of growth. Thinner sutures are used with young children. Rapid absorbable sutures that generate an inflammatory response are to be avoided on exposed areas. On the contrary, intradermal slow absorbable sutures are a good option to avoid marks. Cyanoacrylate 


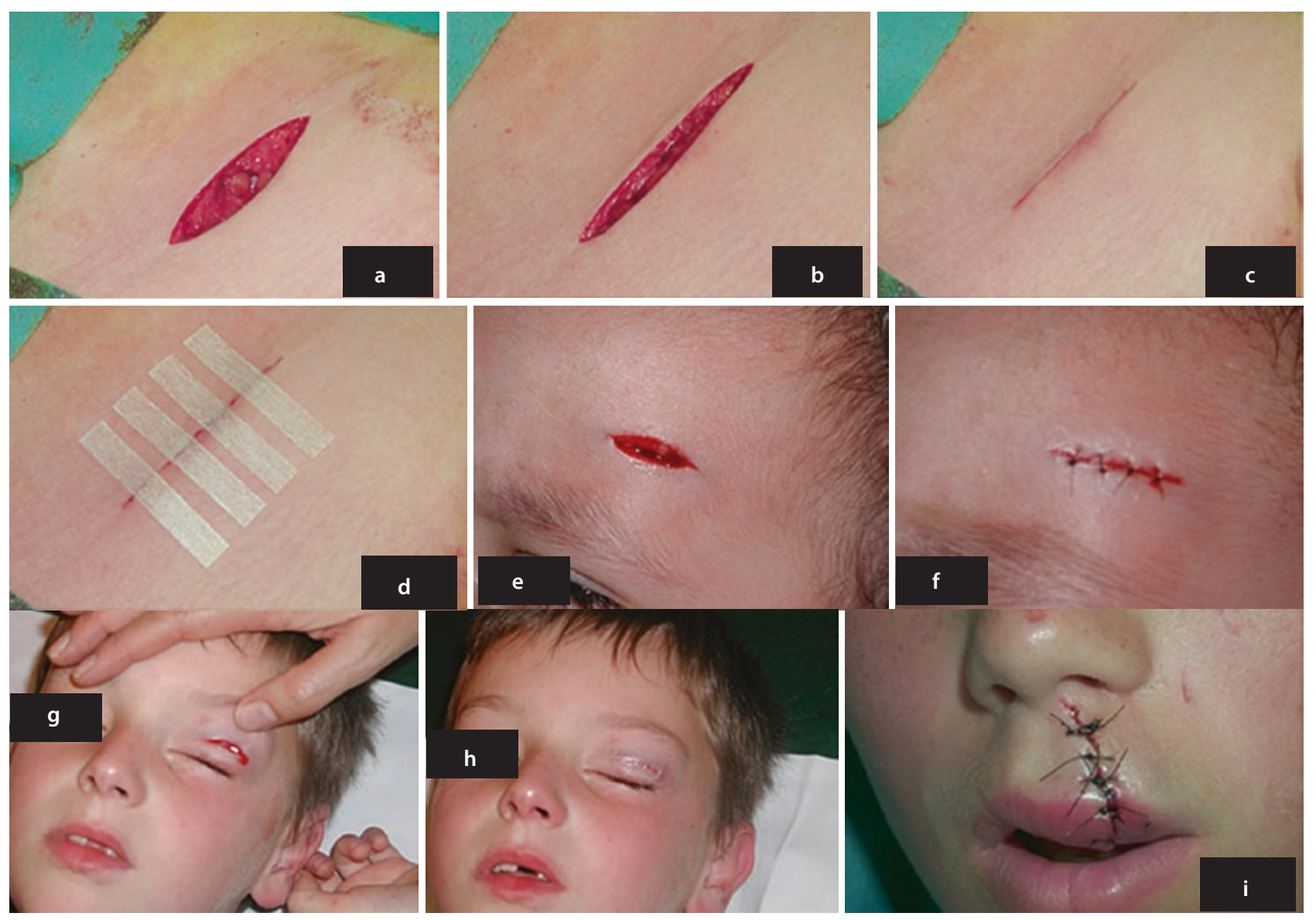

- Fig. 46.1 a-d: Good-quality intradermal suture; $\mathbf{e}$ and $\mathbf{f}$ : good-quality epidermal suture; $\mathbf{g}$ and $\mathbf{h}$ : cyanoacrylate tissue adhesive; i: poorquality epidermal suture, too thick sutures and under wedging

tissue adhesive may be used but is appropriated for very superficial wounds (• Fig. 46.1).

Dressings have to be painless at removal, easy to apply. It is better to realize dressing with hand rather than with forceps because it is quicker and less frightening. Topics should be elementary; healing is rarely a problem in pediatric practice as child's body continuously synthesizes tissue for growth. Therefore, NPWT is most often rapidly effective, preparing wound to be grafted. Flap indications are therefore less frequent in pediatric practice than in adult practice.

\subsection{Pathological Scars}

Scar is the result of the healing process. Ideal scar is thin, flat, white, supple, elastic, and painless, but it exists and is perfectly identifiable under microscope (• Fig. 46.2).

Pathological scars are due to dysregulation of healing phenomena, especially due to the dysregulation that occurs during the modeling phase. This results in prolonged or definitive inflammation and are called hypertrophic or keloid scars [10].

\subsubsection{Clinical and Histological Aspects}

At the beginning of their evolution, it is not possible to distinguish hypertrophic scars from keloid scars: both remain very inflammatory beyond the normal period, red, hot, tense, and itchy. Only the evolution can distinguish them (• Fig. 46.3).

- Hypertrophic scar: despite a marked and prolonged inflammation period, the inflammatory signs will eventually be amended, leading to scar enlargement and fibrosis resulting in a thick scar. Histologically, collagen is abundant, is immature (collagen III in excess), is organized in more flat bundles than in a normal dermis, and contains nodules. Secretion of transforming growth factor $\beta$ (TGF $\beta$ ) and platelet derived growth factor (PDGF) is abundant. Fibroblasts are numerous, responsible for the secretion of collagen in excess. Mast cells are numerous, secreting histamine which is probably responsible for pruritus.

- Keloid scar: evolution goes on forever, leading to extension of lesions beyond the initial limits of the scar, with persistence of inflammatory signs. Sometimes, telangiectasia appears at the surface of the scar. Histologically, 

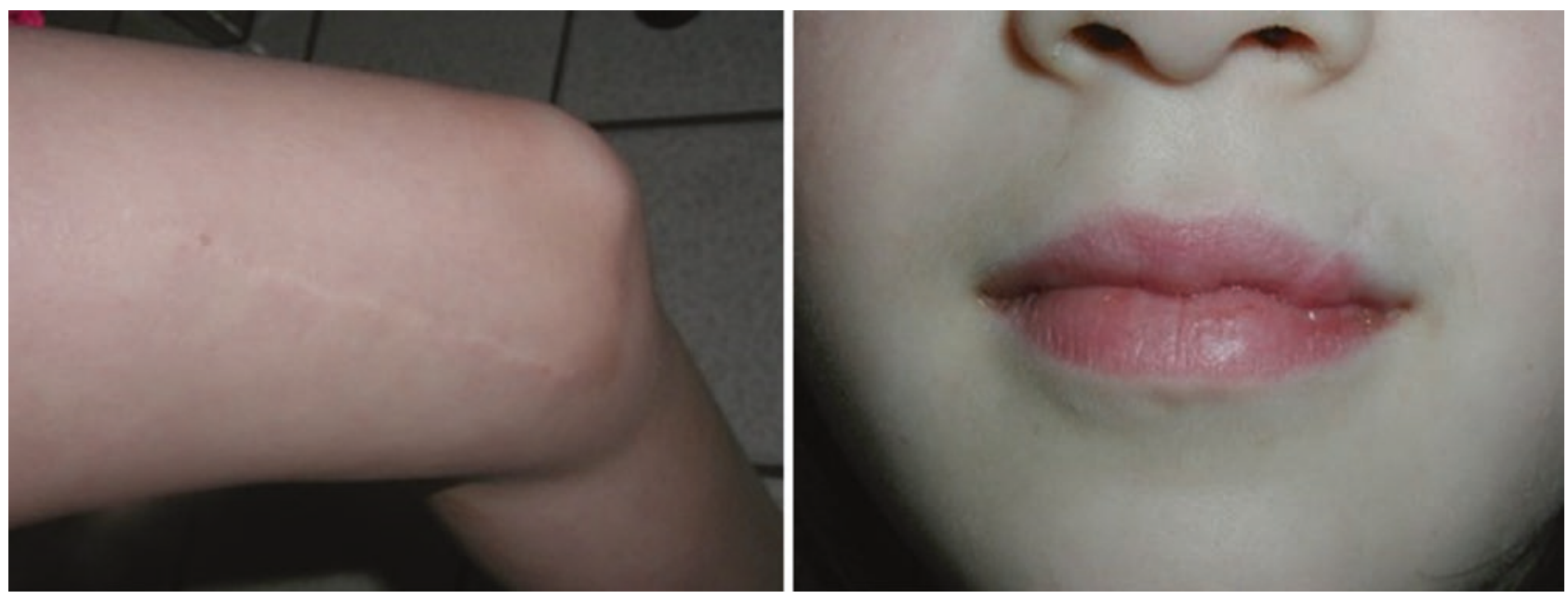

- Fig. 46.2 "Ideal" scars
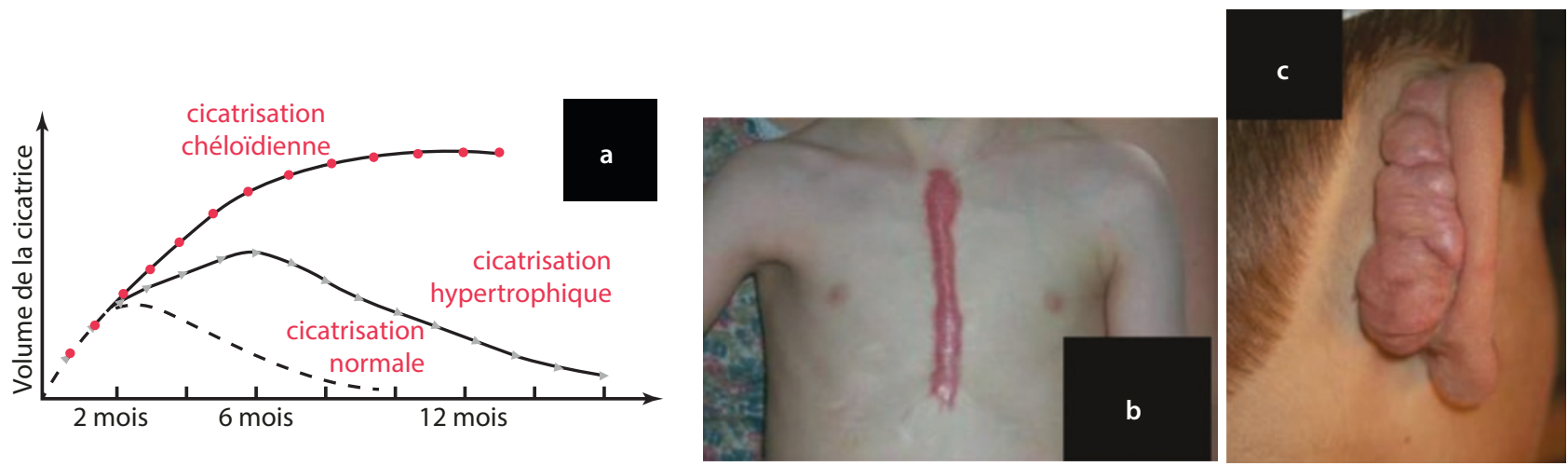

- Fig. 46.3 Pathological scars: a: evolution diagram; b: hypertrophic scar; and c: keloid scar

we found the same characteristics as for hypertrophic scar but with a complete disorganization of the collagen arrangement: there are no more beams but collagen fibers randomly connected and poorly oriented.

\subsubsection{Easing Factors}

Most often, pathological scars occur on a particular field. Although the causal link is not always obvious, there are several factors that contribute to the same scar.

- There is no male or female predominance. The sex ratio for pathological scars is equal to 1 .

- There is no evidence of hereditary factor. However, some authors have questioned about the hereditary characters of keloid scars [11].

- Nevertheless, the ethnic origin seems to be decisive. A preponderance of keloid scars is observed in subjects with pigmented skin (Black, Asian, Métis, etc.). Some studies submit biological hypotheses related to
- The age of the patient is a determining factor. Hypertrophic and keloid scars are exceptional in the elderly and are very common in young people and around puberty. It is therefore essential for pediatric surgeons to be familiar with this scar pathology.

- The location of the wound plays a clear role: the scapular region, the ear area, and the midline of the trunk (presternal and medio-abdominal) are particularly concerned by the development of pathological scars.

- The types of wounds, such as deep burn, soiled wounds, and presence of foreign bodies in the wound, will prolong the cleansing phase and therefore the inflammatory phenomena.

- Hormonal factors: the preponderance of pathological scars at the time of puberty, the impact of pregnancy on scars, and the regression of hypertrophic scars at the time of menopause seem to evoke the role of estrogen. Some studies have looked at the use of antiestrogens in the treatment of hypertrophic scars [12]. 
Although all these factors may have been suggested as favoring pathological scars, only the age, the ethnic factors, and the location of the wound are really the determining factors. Mechanism of occurrence of these pathological scars is not yet totally understood.

\subsubsection{Prevention and Treatment [13]}

Treatment is often disappointing. Many treatments have been proposed, but they are quickly abandoned as they are ineffective. The physio-pathological approach of mechanisms has helped rationalize treatments, particularly by acting on inflammation. Nevertheless, the results often remain below the expectations of the patient and the physician.

Prevention, especially in high-risk population such as pediatric patients, is often effective.

- Prevention of pathological scars

- To be really preventive, treatment must be applied as soon the wound is healed: a few days after primary closure or as soon as epimerization is achieved in secondary closure.

- Reduce inflammation

- Decrease healing time by activating mechanical cleansing.

- Decrease healing time by using surgical means of cover (grafts, flaps).

- Use non-absorbable or slow absorbable intradermal sutures for which inflammatory response is less intense.

- Massage of recent scars reduce edema and mechanically dissociate molecular aggregates. Therefore, massages have a real mechanical action. Massages are most often made with an emollient cream because they are better tolerated. However, massages would be just as effective as without cream.

- Pressure therapy will also reduce edema, inducing a relative tissue hypoxia, thereby minimizing local inflammation. Silicone sheets act in a same way on inflammation [14] (• Fig. 46.4).
- Reduce tension on the scar

- Use Langer's or Borges's lines in surgery.

- Reduce tension on scar by using strips and splinting adjacent joints.

- Treatment of pathological scars

Some treatments are well established, even if their efficacy is disappointing. Other treatments are rather perspectives and subject to current studies. However, the evolution of these scars is sometimes so hopeless for the patient that minimally invasive techniques deserve to be tempted, even if their efficacy is very inconsistent.

Several techniques can be associated either simultaneously or successively.

When a scar becomes inflammatory, the earlier the treatment will be initiated, the more effective it will be.

Preventive techniques can be used as treatment techniques, but they will not be so effective.

- Massages

- Pressure therapy (• Fig. 46.5)

- Silicone sheets

- Steroids have been used for a long time to treat pathologic scars.

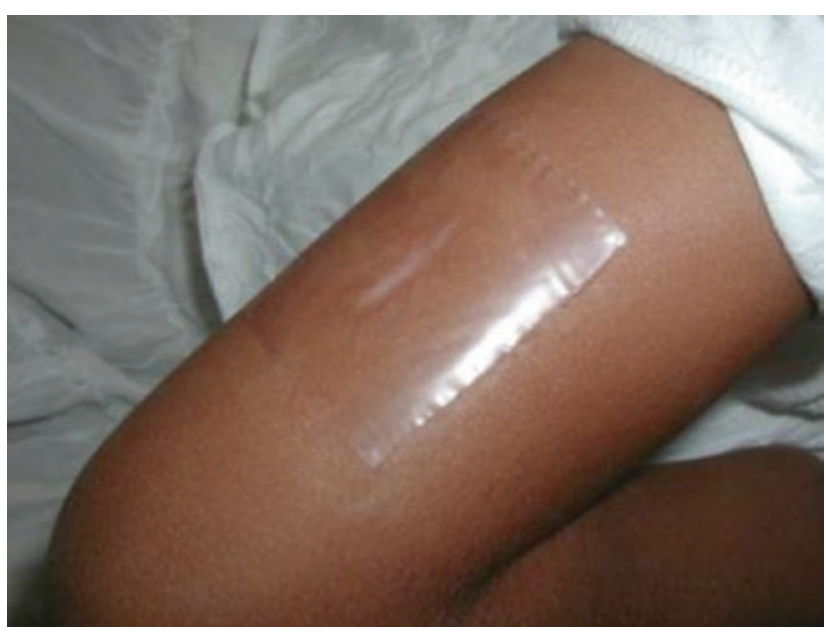

- Fig. 46.4 Silicone sheet on hypertrophic scar
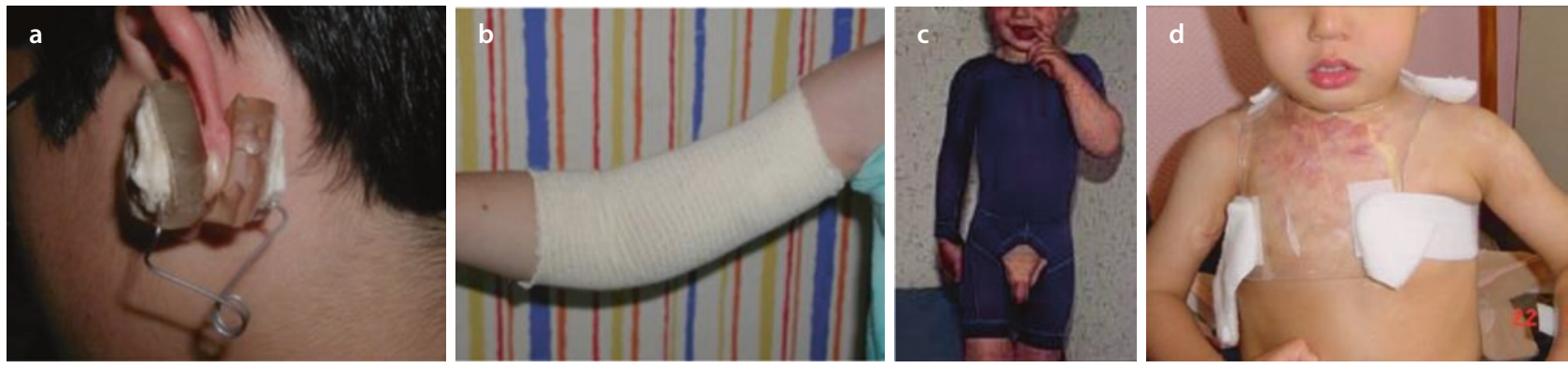

- Fig. 46.5 Different devices for pressure therapy 
They can be used as topic (cream or ointment) or in situ injections.

Local application may be dangerous in children, as the dose of absorbed steroids is difficult to evaluate. An overdose in steroids can lead to a partial or complete Cushing syndrome, which may occur due to abusive local application of corticoids.

For this reason, we prefer local injections of steroids in pediatric practice, using steroids of delayed action with minimal general effects. Injection is proceeded with dermo-jet or syringe and needle after local anesthesia (- Fig. 46.6). Nevertheless, it is often more comfortable to do so under general anesthesia in day surgery unit. Injections can be repeated until at least 2 months between two injections so as not to overload the scar with steroids. The only contraindication is the local infection. Most of the time, flattening of the scar and disappearance of functional signs (pain, pruritus) are observed.

However, it is necessary to be cautious because steroid overload results in epidermal thinning and appearance of telangiectasia.

- Cryotherapy [15] may be used with inconsistent results: it may be used by contact or with freezing needles (• Fig. 46.7).

- Surgery will induce more inflammatory reactions. Therefore, surgery on its own will not solve the prob-

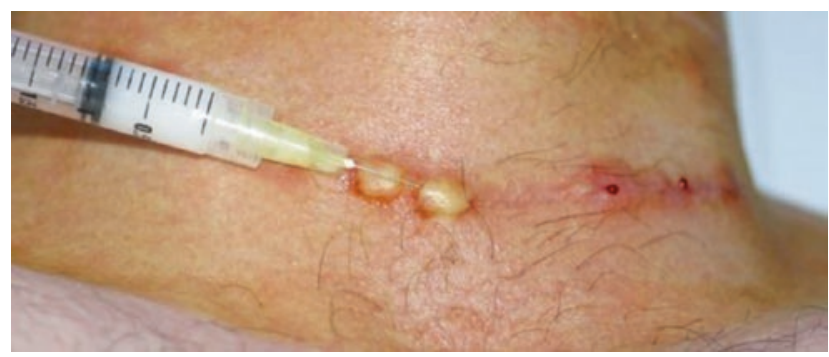

- Fig. 46.6 Intralesional injection of steroids lem: it must be a step in a therapeutic scheme, associating several techniques, and must be proceeded only at the end of the scar maturation, when the inflammatory phenomena have calmed down.

Intralesional excision is the only way to prevent recurrence, but it does not mean that it consistently gives the expected result. The volume of the scar will decrease, which will improve comfort and release functional effects, and perhaps the residual scar will be more receptive to the complementary treatments that are necessary to avoid recurrence.

An injection of delay-steroids can be performed in the dermis of the banks of the excision wound before closing by non-absorbable sutures. Pressure therapy will be prescribed systematically as soon as healing is achieved (10 days).

- Radiations (X-rays, radium, iridium) may be proposed, but they are not to be used in pediatric practice because of carcinogenic risk.

- Anti-neoplastic substances [16] have been the subject of research in recent years: Interferons, mitomycin C, bleomycin, 5-fluorouracil [17] are all molecules that are used as injections in situ, either alone or in combination with corticosteroids. These treatments have to be used very carefully and when there is no other therapeutic possibility in pediatric practice because of their interaction with growth.

- Laser therapy has been tried on pathological scars [18] and is easy to use in pediatric practice under contact local anesthesia.

- Finally, it may be wise, in some situations, to allow time for inflammatory phenomena to decrease spontaneously before initiating aggressive therapies. This implies to support the patient because the evolution often seems hopeless.

Multiple treatments are proposed for these pathological scars due to their uncertain efficacy and the difficulty in achieving results that satisfy the patient (and the physician).
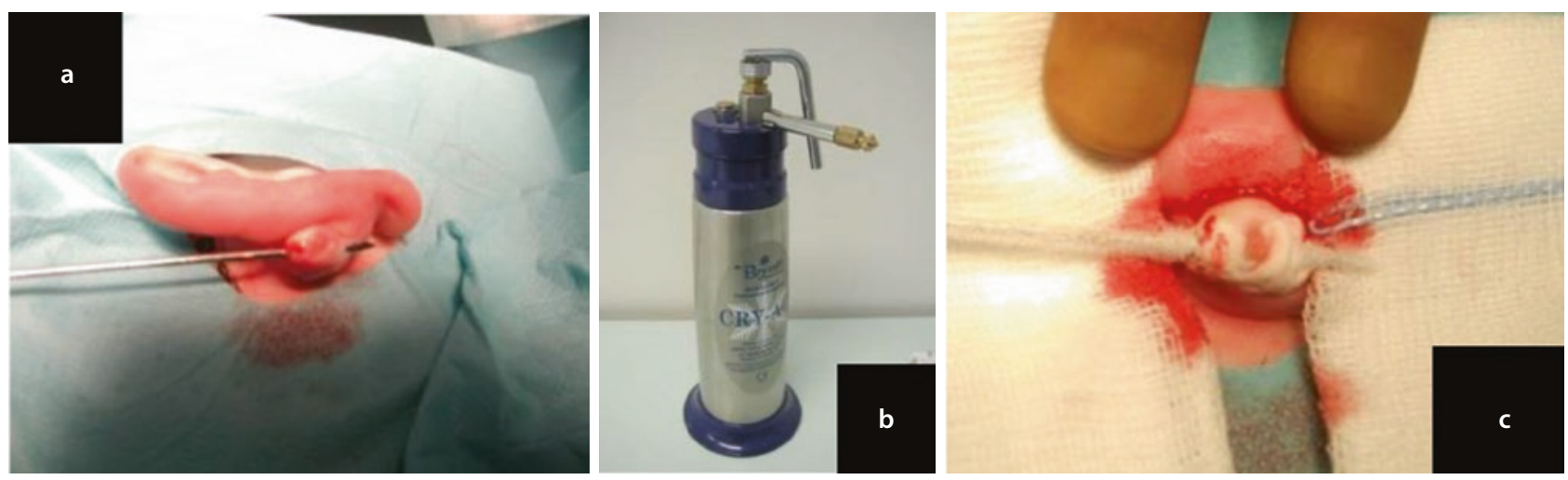

- Fig. 46.7 a-c: Cryotherapy 


\subsection{Defective or Disgracious Scars}

Some scars are defective or disgracious and do not disturb the healing process: hypotrophic or enlarged scars, adherent scars, dyschromic scars, tattooed scars, granuloma, technical defects, etc.

All scars that present defects but no pathology of the healing process can be corrected by surgery, irrespective of the technique used: surgical recovery, adipocytes transplantation (Coleman technique), dermabrasion with or without vaporization of autologous keratinocytes, etc. However, it will be necessary to wait at least until the end of the maturation phase to consider secondary surgery.

Deciding what is the best time to practice this surgery is sometimes difficult in pediatric practice. Apart from functional indications for which the question hardly arises, the indications are most often aesthetic. It is then necessary to ensure the child's actual motivations and that this is not a parental request.

If the child is voluntary, a "contract" must then be passed with the child or teenager emphasizing three key points:

- The disgracious scar will be replaced by a scar that hopefully will be of better quality. There is no magic rubber for scar.

- Postoperative scar management (e.g., strips, silicone, possible splint, and refraining from sportive activities) is constraining but necessary for a good result.

- If the patient is not able to undergo scar management, surgery is useless.

All these scar anomalies can be corrected, but it is of course essential to prevent and avoid them.

\subsection{Scars and Growth}

During the first 2 years after healing, the scar matures. Contraction of the scar is current and normal due to the action of myofibroblasts. After this period of time, the scar is quiet and more or less definitive.

In pediatric practice, the patient will grow with the scar. This may induce tractions because the scar is fibrous and does not grow as much as the patient. If this is not corrected, it may induce bending because of asymmetric growth.

Psychomotor development may be disturbed by scars. Physicians should be aware that psychological impact of scars evolves over time all along childhood and teenage. Therefore, pediatric patients with scars have to be followed all along their growth period to detect the need for scar surgery, whether it is for functional purpose or for aesthetic and psychological purpose.

\subsection{Conclusion}

Healing physiology is not different in children but the importance of inflammatory mechanisms makes the resulting scar different. Hypertrophic scar is physiological in pediatric practice and has to be prevented.

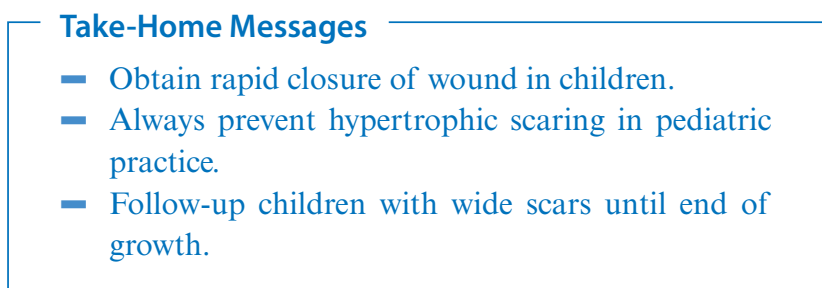

\section{References}

1. Longaker MT, Adzick NS. The biology of fetal wound healing: a review. Plast Reconstr Surg. 1991;87:788-98.

2. Hopkinson-Woolley J, Hughes D, Gordon S, Martin P. Macrophage recruitment during limb development and wound healing in the embryonic and foetal mouse. J Cell Sci. 1994;107:1159-67.

3. Oztürk S, Deveci M, Sengezer M, Günhan Ö. Results of artificial inflammation in scarless foetal wound healing: an experimental study in foetal lambs. Br J Plast Surg. 2001;54:47-52.

4. Kishi K, Nakajima H, Tajima S. Differential responses of collagen and glycosaminoglycan syntheses and cell proliferation to exogenous transforming growth factor beta 1 in the developing mouse skin fibroblasts in culture. Br J Plast Surg. 1999;52(7): 579-82.

5. Longaker MT, Adzick NS, Hall JL, Stair SE, Crombleholme TM, Du BW, Bradley SM, Harrisson MR, Stern R. Studies in fetal wound healing, VII. Fetal wound healing may be modulated by hyaluronic acid stimulating activity in amniotic acid. $\mathrm{Br}$ J Plast Surg. 1990;25:430-3.

6. Longaker MT, Whitby DJ, Ferguson MW, Lorenz HP, Harrisson MR, Adzick NS. Adult skin wounds in the fetal environment heal with scar formation. Ann Surg. 1994;219:65-72.

7. Le Touze A. Cicatrisation normale et pathologique. In: Captier G, editor. Chirurgie plastique de l'enfant et de l'adolescent. Montpellier: Sauramps Medical; 2015.

8. Jeffs D, Dorman D, Brown S, Files A, Graves T, Kirk E, Meredith-Neve S, Sanders J, White B, Swearingen CJ. Effect of virtual reality on adolescent pain during burn wound care. J Burn Care Res. 2014;35(5):395-408.

9. Burns-Nader S, Joe L, Pinion K. Computer tablet distraction reduces pain and anxiety in pediatric burn patients undergoing hydrotherapy: a randomized trial. Burns. 2017;43(6):1203-11.

10. Lee HJ, Jang YJ. Recent understandings of biology, prophylaxis and treatment strategies for hypertrophic scars and keloids. Int $\mathrm{J}$ Mol Sci. 2018;19(3):711.

11. Marneros AG, Norris JE, Olsen BR, Reichenberger E. Clinical genetics of familial keloids. Arch Dermatol. 2001;137(11): 1429-34.

12. Gragnani A, Warde M, Furtado F, Ferreira LM. Topical tamoxifen therapy in hypertrophic scars or keloids in burns. Arch Dermatol Res. 2010;302(1):1-4.

13. Monstrey S, Middelkoop E, Vranckx JJ, Bassetto F, Ziegler UE, Meaume S, Téot L. Updated scar management practical guide- 
lines: non-invasive and invasive measures. J Plast Reconstr Aesthet Surg. 2014;67(8):1017-25.

14. O'Brien L, Jones DJ. Silicone gel sheeting for preventing and treating hypertrophic and keloid scars. Cochrane Database Syst Rev. 2013;(9):CD003826.

15. O'Boyle CP, Shayan-Arani H, Hamada MW. Intralesional cryotherapy for hypertrophic scars and keloids: a review. Scars Burn Heal. 2017;3:2059513117702162.

16. Shridharani SM, Magarakis M, Manson PN, Singh NK, Basdag $\mathrm{B}$, Rosson GD. The emerging role of antineoplastic agents in the treatment of keloids and hypertrophic scars: a review. Ann Plast Surg. 2010;64(3):355-61.

17. Ren Y, Zhou X, Wei Z, Lin W, Fan B, Feng S. Efficacy and safety of triamcinolone alone and combination with 5-fluorouracil for treating hypertrophic scars and keloids: a systemic review and meta-analysis. Int Wound J. 2017;14:480-7.

18. Jin R, Huang X, Li H, Yuan Y, Li B, Cheng C, Li Q. Laser therapy for prevention and treatment of pathologic excessive scars. Plast Reconstr Surg. 2013;132(6):1747-58.

Open Access This chapter is licensed under the terms of the Creative Commons Attribution 4.0 International License (http://creativecommons. org/licenses/by/4.0/), which permits use, sharing, adaptation, distribution and reproduction in any medium or format, as long as you give appropriate credit to the original author(s) and the source, provide a link to the Creative Commons license and indicate if changes were made.

The images or other third party material in this chapter are included in the chapter's Creative Commons license, unless indicated otherwise in a credit line to the material. If material is not included in the chapter's Creative Commons license and your intended use is not permitted by statutory regulation or exceeds the permitted use, you will need to obtain permission directly from the copyright holder.

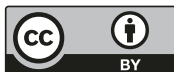

